\title{
Other incarnations of the Gross-Pitaevskii dark soliton
}

\author{
Indubala I. Satija ${ }^{\mathrm{a}, \mathrm{b}}$, Radha Balakrishnan ${ }^{\mathrm{c}, *}$ \\ a Department of Physics, George Mason University, Fairfax, VA 22030, United States \\ b National Institute of Standards and Technology, Gaithersburg, MD 20899, United States \\ c The Institute of Mathematical Sciences, Chennai 600113, India
}

\section{A R T I C L E I N F O}

\section{Article history:}

Received 22 June 2010

Accepted 6 December 2010

Available online 8 December 2010

Communicated by A.R. Bishop

\section{Keywords:}

Soliton

Bose-Einstein condensate

Bose-Hubbard model

Hard-core boson

Magnetic soliton

\begin{abstract}
A B S T R A C T
We show that the dark soliton of the Gross-Pitaevskii equation (GPE) that describes the Bose-Einstein condensate (BEC) density of a system of weakly repulsive bosons, also describes that of a system of strongly repulsive hard core bosons at half filling. As a consequence of this, the GPE soliton gets related to the magnetic soliton in an easy-plane ferromagnet, where it describes the square of the in-plane magnetization of the system. These relationships are shown to be useful in understanding various characteristics of solitons in these distinct many-body systems.
\end{abstract}

(C) 2010 Elsevier B.V. All rights reserved.

\section{Introduction}

The study of solitary waves and solitons has been one of the most active areas of research in nonlinear systems for the past three decades [1]. As is well known, a solitary wave is a spatially localized traveling wave which maintains its shape, size and speed as it travels. If a solitary wave retains all its features even after a collision with another solitary wave, it is called a soliton. Some of the iconic examples of completely integrable nonlinear partial differential equations arising in applied mathematics and exhibiting such strict soliton solutions [2] include the Korteweg-de Vries equation, the sine Gordon equation, the nonlinear Schrödinger equation, etc. However, in physics literature a solitary wave itself is often referred to as a soliton due to its particle-like properties and we will do so in this Letter as well. Solitons appear in diverse fields [1] such as hydrodynamics, fiber optics, biophysics, high energy physics, magnetism and in various condensed matter systems including a Bose-Einstein condensate (BEC). In addition to theoretical investigations, they have also been observed experimentally in various nonlinear systems.

A BEC is an intrinsically nonlinear system. The Gross-Pitaevskii equation (GPE) [3] has been central to explaining various fundamental aspects of the BEC of a system of weakly repulsive bosons. One of the hallmarks of this system is the dark soliton that has been theoretically predicted [4] as a unidirectional traveling wave solution for the condensate density. This soliton, which dies out

\footnotetext{
* Corresponding author.

E-mail address: radha@imsc.res.in (R. Balakrishnan).
}

when its speed approaches the speed of sound, has also been observed experimentally [5]. The study of solitary wave propagation in BEC remains an active frontier with emphasis on non-GPE dynamics [6] and many body effects such as quantum fluctuations and depletion that cannot be described by GPE $[7,8]$.

In view of the relatively few examples of systems exhibiting solitons, any relationship that is found between different physical systems is fascinating, since it unveils the universal mathematical aspects underlying these nonlinear systems that describe completely different physical phenomena. In this Letter, we show that the dark soliton of the GPE that describes the BEC density of a system of weakly repulsive bosons, also describes that of a system of strongly repulsive hard core bosons at half filling. This in turn leads to a connection between the GPE soliton and a magnetic soliton in an easy-plane ferromagnet. These relationships are shown to be useful in understanding various characteristics of solitons in these distinct many-body systems.

The complex order parameter (i.e., the condensate wave function) describing a BEC that satisfies the GPE can be identified with the bosonic coherent state average [9] of the boson annihilation operator in the continuum version of the Bose-Hubbard model. Analogously, in a strongly interacting limit described by a system of hard-core bosons (HCB) the appropriate order parameter for this system would be the spin coherent state average [10] as the system maps to a pseudospin model [11]. In the continuum description of the dynamics of the extended lattice Bose-Hubbard model for hard-core bosons with nearest neighbor interactions, this order parameter satisfies an evolution equation that is different from the GPE [12]. We will refer to this equation as the HGPE, where the prefix "H" has been used to denote its connection to HCB. 
Unlike the GPE which describes the dynamics of a BEC with no depletion, the HGPE evolution encodes both the normal and condensate fractions in the Bose system. In our recent study, we showed that the HGPE supports [12] both a dark soliton and an antidark [13] soliton (i.e., a bright soliton on a pedestal) for the bosonic density. In other words, the dark soliton of the BEC of weakly repulsive bosons acquires a partner that is bright, when the repulsion becomes extremely strong. Apart from being bright, the antidark soliton is found to be of quite a distinct variety compared to the dark soliton that resembles the GPE soliton. Away from half-filling, i.e., when there is a nonvanishing particle-hole imbalance in the background, the dark soliton dies out when its propagation speed approaches the speed of sound (like the GPE soliton), while its brighter partner is found to persist all the way up to the sound velocity [12].

In contrast, at half-filling, when the particle-hole imbalance is zero, the dark and the antidark solitons in the particle density profile of the HGPE become mirror images of each other, and they both die out at the sound velocity. Interestingly, in the corresponding condensate density, the two solitons become degenerate and cease to exist as separate entities. In this special case, the condensate density profile is a dark soliton that dies out as its speed approaches the speed of sound [12].

In this Letter, we show that for the half-filled case, the localized functional form of the solitary wave solution for the density of the hard-core bosonic condensate described by the HGPE agrees with that of the dark soliton of the GPE, to a very good approximation. In other words, if one focuses on the condensate fraction of the hard-core bosonic system, this strongly repulsive Bose gas supports a solitary wave that has almost the same profile as the GPE soliton that describes the BEC of a weakly repulsive Bose gas.

Furthermore, since the HCB gas in the spin coherent state formulation of the extended Bose-Hubbard model with nearest neighbor interactions mimics an anisotropic, easy-plane ferromagnetic chain, the well-known dark soliton of the GPE also relates to the magnetic soliton in the easy-plane ferromagnet, where it describes the profile of the square of the in-plane magnetization.

In Section 2, we begin with the many-body Bose-Hubbard Hamiltonian that describes the low-energy behavior of bosonic atoms in an optical lattice, and briefly outline some steps that lead to the order parameter equations in the weakly and strongly interacting limits, respectively. In Sections 3 and 4, we first derive the evolution equation for the traveling waves in the condensate fraction of the atomic cloud obtained from the HGPE, and show that to a very good approximation, this equation agrees with the corresponding equation derived from the GPE. In Section 5, we discuss the relationship between the density solitons of the BEC and the magnetic solitons of an easy-plane ferromagnet.

\section{The Bose-Hubbard model, the GPE, and the HGPE}

We begin with the extended lattice Bose-Hubbard model in $d$ dimensions, whose Hamiltonian is given by

$$
\begin{aligned}
H= & -\sum_{j, a}\left[t b_{j}^{\dagger} b_{j+a}+V n_{j} n_{j+a}\right] \\
& +\sum_{j} U n_{j}\left(n_{j}-1\right)-(\mu-2 t) n_{j},
\end{aligned}
$$

where $b_{j}^{\dagger}$ and $b_{j}$ are the creation and annihilation operators for a boson at the lattice site $j, n_{j}$ is the number operator, $a$ labels nearest-neighbor $(\mathrm{nn})$ separation, $t$ is the nn hopping parameter, $U$ is the on-site repulsion strength, and $\mu$ is the chemical potential. To soften the effect of strong onsite repulsion, we add an attractive $\mathrm{nn}$ interaction $(V>0)$. Such a term may mimic certain characteristics of the long range dipole-dipole interaction, that has been considered in several recent studies [14]. The term $2 t n_{j}$ is added so that the terms involving $t$ reduce to the kinetic energy expression in the continuum version of the many-body bosonic Hamiltonian.

Conventionally, one defines the order parameter for a many boson system to be the thermodynamic expectation value of the boson field operator. Invoking the concept of a broken gauge symmetry allows this expectation value to be nonzero below the BEC transition temperature. It has been argued [9] that this order parameter may be chosen to be the expectation value of the boson annihilation operator in the bosonic coherent state (also known as the harmonic oscillator coherent state or Glauber coherent state) representation of the pure quantum state. In this description, it can be shown that quantum fluctuations are absent.

The Heisenberg equation of motion for the boson annihilation operator determined from (1), after a bosonic coherent state averaging yields the following GPE equation for the condensate order parameter $\Psi_{g}(\mathbf{r}, t)$ in the continuum description

$-\left(\hbar^{2} / 2 m\right) \nabla^{2} \Psi_{g}+U\left|\Psi_{g}\right|^{2} \Psi_{g}-\mu \Psi_{g}=i \hbar \partial_{t} \Psi_{g}$.

The GPE with $U>0$ provides a very useful characterization of the various properties of the $\mathrm{BEC}$ of weakly repulsive bosons [3]. Here the condensate density $\rho_{g}=\left|\Psi_{g}\right|^{2}$ satisfies a continuity equation, and the system shows no depletion.

The HCB limit $(U \rightarrow \infty)$ of the Bose-Hubbard Hamiltonian (1) has emerged as a useful model to describe various characteristics of the BEC of a strongly repulsive Bose system. The constraint that two bosons cannot occupy the same site can be incorporated in the formulation by using field operators that anticommute at the same site but commute at different sites, thus satisfying the same algebra as that of a spin- $\frac{1}{2}$ system. By identifying $b_{j}$ with the spin flip operator $\hat{S}_{j}^{+}$, along with $n_{j}=\hat{S}_{j}^{+} \hat{S}_{j}^{-}=\frac{1}{2}-\hat{S}_{j}^{z}$, the system can be mapped to the following quantum XXZ Hamiltonian in a magnetic field:

$H_{S}=-\sum_{j, a}\left[t \hat{\mathbf{S}}_{j} \cdot \hat{\mathbf{S}}_{\mathbf{j}+\mathbf{a}}-g \hat{S}_{j}^{z} \hat{S}_{j+a}^{z}\right]-\sum_{j}(g-\mu) \hat{S}_{j}^{z}$.

Here $g=(t-V) d$, where $d$ is the spatial dimensionality. As we shall show, $g=(t-V)>0$ (see below Eq. (8)). Hence the HCB system maps to a quantum spin-1/2 system with a Heisenberg exchange interaction $t$, an easy-plane exchange anisotropy $g$ and a transverse magnetic field $h_{z}=(g-\mu)$ along the $z$-direction.

This mapping to spins suggests that a natural choice for the condensate order parameter $\Psi_{s}$ of the HCB system is the average of the spin flip operator in the spin coherent state representation [15], i.e.,

$\Psi_{S}=\left\langle\hat{S}^{+}\right\rangle$.

In this representation, it can be shown that [12] the condensate density, $\rho_{s}=\left|\Psi_{s}\right|^{2}=\left\langle\hat{S}^{-}\right\rangle\left\langle\hat{S}^{+}\right\rangle$is related to total particle number density $\rho=\left\langle\hat{S}^{-} \hat{S}^{+}\right\rangle$as

$\rho_{s}=\rho(1-\rho)$.

Setting $\Psi_{s}=\sqrt{\rho_{s}} \exp (i \phi)$ in the continuum description, the condensate order parameter $\Psi_{s}$ and the particle density $\rho$ satisfy the following equations [12]

$$
\begin{aligned}
& i \hbar \dot{\Psi}_{s}=-\frac{\hbar^{2}}{2 m}(1-2 \rho) \nabla^{2} \Psi_{s}-V_{e} \Psi_{s} \nabla^{2} \rho+2 g \rho \Psi_{s}-\mu \Psi_{s}, \\
& \dot{\rho}=\frac{\hbar}{2 m} \nabla \cdot[\rho(1-\rho) \nabla \phi],
\end{aligned}
$$

with the identification $t a^{2}=\hbar^{2} / m$ and $V a^{2}=V_{e}$. Eq. (6) (which can also be written as coupled equations for $\rho$ and $\phi$ ) will be re- 
ferred to as HGPE. Using the asymptotic value $\rho \rightarrow \rho^{0}$ in Eq. (6), the chemical potential $\mu$ is found to be $\mu=2 g \rho^{0}$.

From Eq. (5), we note that it is important to distinguish between the total bosonic particle density $\rho$ and the condensate density $\rho_{s}$, where $\rho=\rho_{s}+\rho_{d}$. Here, $\rho_{d}$ describes the depletion, i.e., the normal component of $\rho$.

Linearizion of HGPE by considering small amplitude fluctuations in the asymptotic value of $\Psi_{s}$ leads to a Bogoliubov-like spectrum. For small momenta, the spectrum becomes linear, leading to the sound velocity $c_{s}$ for the hard-core system as

$c_{s}=\left(2 g \rho_{s}^{0} / m\right)^{\frac{1}{2}}$,

where $\rho_{s}^{0}$ is the asymptotic value of the condensate density. Since $c_{s}$ must be real, we obtain the condition $g=(t-V)>0$.

In the next section, we will derive an equation of motion for the condensate density $\rho_{s}$ that arises from HGPE, for the half-filled case, when the asymptotic particle density is half, i.e., when the number of particles equals the number of holes in the background.

\section{HCB condensate density evolution in the half-filled case of HGPE}

By seeking unidirectional traveling wave solutions (along the $x$ direction) of the total density $\rho$ of the form,

$\rho(z)=\rho_{0}+f(z)$

where $z=(x-v t) / a$ and $\rho_{0}$ denotes the constant asymptotic value of the background density, it has been shown [12] that the HGPE (Eq. (6)) leads to a nonlinear differential equation for $f(z)$ which can be solved analytically to a very good approximation, to yield soliton solutions.

Here we focus on the half-filled case with $\rho_{0}=1 / 2$. For this case, the differential equation for $f$ takes the form [12]

$\left(\frac{d f}{d \bar{z}}\right)^{2}=4 f^{2}\left[\gamma^{2}-f^{2}\right]$.

Here, $\gamma^{2}=1-\bar{v}^{2}$ with $\bar{v}=v / c_{s}$. Further, $\bar{z}=\zeta z$ and $\zeta=$ $\Lambda / \sqrt{1-2 \Lambda^{2}}$, where the microscopic dimensionless parameter $\Lambda=c_{s} / c_{0}$ is the speed of sound $c_{s}$ in the HCB condensate measured in units of the zero-point velocity $c_{0}=\hbar / \mathrm{ma}$.

As discussed in [12], Eq. (10) provides the following two soliton solutions

$f(\bar{z})= \pm(\gamma / 2) \operatorname{sech}(2 \gamma \bar{z})= \pm(\gamma / 2) \operatorname{sech}\left(z / \Gamma_{s}\right)$,

where the soliton width $\Gamma_{s}=(2 \gamma \zeta)^{-1}$. Since $\gamma$ must be real in Eq. (11), the dimensionless parameter $\bar{v}$ must satisfy $0<\bar{v}<1$.

The two solutions for $f$ in (11) lead to a doublet of localized solitons, namely, a dark soliton and an antidark soliton, for the density of the bosonic cloud $\rho(\bar{z})=(1 / 2)+f(\bar{z})$. Using this in the condensate density expression given in Eq. (5), we get

$\rho_{s}(\bar{z})=\frac{1}{4}-f^{2}(\bar{z})$.

Writing $\rho_{S}(\bar{z})=(1 / 4)+f_{S}(\bar{z})$, and comparing it with Eq. (12) shows that the variation $f_{s}$ around the asymptotic condensate density and the variation $f$ around the total density $\rho$ are related as follows:

$f_{s}(\bar{z})=\rho_{s}(\bar{z})-\frac{1}{4}=-f^{2}(\bar{z})$.

Differentiating both sides of Eq. (13) with respect to $\bar{z}$ and using Eq. (10) in the resulting equation, we obtain the following differential equations for the normalized condensate density $\bar{\rho}_{s}=\rho_{s} /(1 / 4)$

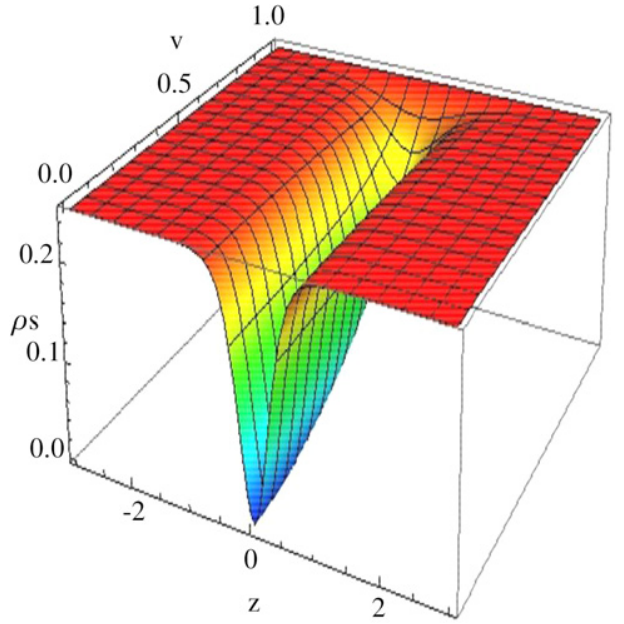

Fig. 1. (Color online.) Condensate density profile $\rho_{s}$ (Eq. (12)) of HGPE at half filling with $V / t=1 / 3$, showing the behavior of the dark soliton as its propagation speed $\bar{v}=v / c_{s}$ increases from 0 to 1 . Note that the soliton flattens out at $\bar{v}=1$.

and the corresponding normalized variation $\bar{f}_{s}=f_{s} /(1 / 4)$, respectively

$\left(\frac{d \bar{\rho}_{s}}{d \bar{z}}\right)^{2}=\left(1-\bar{\rho}_{s}\right)^{2}\left(\bar{\rho}_{s}-\bar{v}^{2}\right)$,

$\left(\frac{d \bar{f}_{s}}{d \bar{z}}\right)^{2}=\bar{f}_{s}^{2}\left(\bar{f}_{s}+\gamma^{2}\right)$

Unlike the doublet soliton solutions of $f$ given in Eq. (11), $\bar{f}_{S}$ has the unique solution

$\bar{f}_{s}=-4 f^{2}=-\gamma^{2} \operatorname{sech}^{2} 2 \gamma \bar{z}=-\gamma^{2} \operatorname{sech}^{2} z / \Gamma_{s}$.

Fig. 1 shows the dark soliton profiles for the condensate density for various values of $\bar{v}$. As the plot shows, the soliton which is dark when $\bar{v}=0$, dies out as $\bar{v} \rightarrow 1$, i.e., as its propagation speed approaches the speed of sound.

\section{Condensate density evolution in the GPE}

In this section, we show that Eq. (14), which describes the evolution of the condensate density of the HGPE for a half-filled system, has the same form as the condensate density evolution equation obtained from the GPE.

Following the earlier studies of solitons in GPE [3], we write the condensate wave function $\Psi_{g}$ in that system as

$\Psi_{g}=\sqrt{\rho_{g}^{0}}\left(\psi_{r}+i \bar{v}\right)$

where $\rho_{g}^{0}$ is the asymptotic value of the background density. $\psi_{r}$ denotes the real part of $\Psi_{g}$. Its imaginary part is $\bar{v}=v / c_{g}$ is the soliton propagation speed measured in units of the sound speed $c_{g}$ for the GPE. It is given by

$c_{g}=\left(U \rho_{g}^{0} / m\right)^{\frac{1}{2}}$.

In Eq. (17), $0<\bar{v}<1$. Further, it can be shown that $\psi_{r}$ satisfies [3]

$\sqrt{2} \frac{d \psi_{r}}{d w}-\left(\gamma^{2}-\psi_{r}^{2}\right)=0$

where $\gamma^{2}=1-\bar{v}^{2}$ and $w=(x-v t) / \xi$, where the healing length $\xi$ is given by the well-known expression $\xi=\hbar / \sqrt{2 m U \rho_{s}^{0}}=$ $\hbar / \sqrt{2} m c_{g}$. 
In analogy with the HGPE (see below Eq. (10)), we define a dimensionless parameter $\Lambda=c_{g} / c_{0}$, which is the sound velocity in the GPE measured in units of the zero-point velocity. This enables us to write $\xi$ in terms of $\Lambda$ as

$\xi=a / \sqrt{2} \Lambda$.

Hence $w=\sqrt{2} \Lambda(x-v t) / a=\sqrt{2} \Lambda z$. Using this in Eq. (19) and squaring both sides of this equation, we get

$\left(\frac{d \psi_{r}}{d \bar{z}}\right)^{2}=\left(1-\psi_{r}^{2}-\bar{v}^{2}\right)^{2}$

where $\bar{z}=\Lambda z$.

Now, from Eq. (17), the normalized condensate density $\bar{\rho}_{g}=$ $\rho_{g} / \rho_{g}^{0}$ for the GPE is

$\bar{\rho}_{g}=\psi_{r}^{2}+\bar{v}^{2}$.

Differentiating Eq. (22) with respect to $\bar{z}$, and using Eq. (21), it is easy to show that $\bar{\rho}_{g}$ satisfies the following equation

$\left(\frac{d \bar{\rho}_{g}}{d \bar{z}}\right)^{2}=\left(1-\bar{\rho}_{g}\right)^{2}\left(\bar{\rho}_{g}-\bar{v}^{2}\right)$.

The above equation describes the spatial evolution of the condensate density whose order parameter obeys the GPE, in a frame moving with velocity $v$. This equation is identical in form to Eq. (14) for the half-filled HGPE, and hence the condensate density $\rho_{g}$ for the GPE (which is equal to the particle density) has the same functional form as the condensate density $\rho_{s}$ for the HGPE. We would like to emphasize that this mapping between the GPE and the HGPE is valid provided one considers only the condensate fraction of the HCB. Since the half-filling case for particle density in HGPE corresponds to quarter-filling for the condensate density, the corresponding GPE density for this mapping to be applicable is $\rho_{g}^{0}=1 / 4$.

The mapping between the GPE and the HGPE discussed above provides the relationship that the width of the soliton for the condensate density in the GPE and the HGPE are given, respectively, by $\Gamma_{g}=(2 \gamma \Lambda)^{-1}$ and $\Gamma_{S}=\left(2 \gamma \Lambda / \sqrt{1-2 \Lambda^{2}}\right)^{-1} .1$

We would like to note that the relationship between the GPE and the HGPE applies only to the condensate density profiles, as the solitons for the corresponding condensate wave functions in these two limiting cases will differ in their phase jumps.

\section{The BEC soliton and the magnetic soliton in the easy-plane ferromagnet}

An interesting consequence of the mapping between the GPE and the HGPE discussed in the previous section is the emergence of a relationship between two distinct physical systems, the GPE soliton in a BEC and a magnetic soliton that arises from the quantum easy-plane ferromagnet (3). As we discuss below, this provides an interesting way to understand why there are in general two solitary waves in the bosonic density of the HCB system, and why in the special case of half-filling, the condensate part of the HCB has only one solitary wave and why it is dark.

In magnetism, the study of strict solitons and solitary waves in various types of quasi-one-dimensional classical spin systems has been a very active field $[16,17]$ for over three decades. In the

\footnotetext{
1 In this relationship, it should be noted that the dimensionless parameter $\Lambda$ is $c_{g} / c_{0}$ for the GPE and $c_{s} / c_{0}$ for the HGPE. Likewise, in the parameter $\gamma=(1-$ $\left.\bar{v}^{2}\right)^{1 / 2}$, the dimensionless soliton speed $\bar{v}$ is $v / c_{g}$ for the GPE and $v / c_{s}$ for the HGPE.
}

present work, the relationship between the Bose-Hubbard model and a quantum ferromagnetic Heisenberg spin Hamiltonian (3) with an exchange anisotropy and a magnetic field will be exploited to relate the BEC soliton to the magnetic soliton. Note that the anisotropy is of the easy-plane type since $g>0$.

By writing the complex parameter $\tau$ appearing in the spin coherent representation [15] as $\tau=\tan (\theta / 2) \exp (i \phi)$, with $0 \leqslant \theta \leqslant \pi$ and $0 \leqslant \phi \leqslant 2 \pi$, the coherent state average $\left\langle\hat{S}^{+}\right\rangle$in the quantum spin- $1 / 2$ system can be calculated in terms of $\theta$ and $\phi$. By identifying $\rho_{s}=\left|\left\langle\hat{S}^{+}\right\rangle\right|^{2}$ we obtain the following relation between the condensate density $\rho_{s}$ and the classical spin variables: $S_{x}, S_{y}, S_{z}$, where $\theta$ and $\phi$ appear as the polar and azimuthal angles of a classical spin vector $\mathbf{S}$.

$\rho_{s}=\left(S_{x}^{2}+S_{y}^{2}\right) / 4=\left[(1 / 4)-S_{z}^{2}\right] / 4$.

In addition, the particle density $\rho$, the chemical potential $\mu$ and the particle-hole imbalance variable $\left(1-2 \rho^{0}\right)$ in the BEC system described by HGPE are related to the spin variables of the magnetic system as follows

$\rho=1 / 2-S_{z}$

$\mu=2 g \rho^{0}=g\left(1-2 S_{z}^{0}\right)$,

$g\left(1-2 \rho^{0}\right)=(g-\mu)=h_{z}$,

where $h_{z}$ is the magnetic field along the $z$-direction, as seen from the XXZ Hamiltonian (3). Of the above, the first equation is obtained by taking the expectation value of the corresponding hardcore boson operator to be spin coherent state average. The last two are obtained by using their asymptotic relationships in the expression for $\mu$ (see below Eq. (7)).

On taking spin-coherent state averages of the quantum spins on the lattice and going to the continuum, the evolution equation for the condensate order parameter also describes the evolution of classical spins, where the asymptotic density controls the external transverse field in the spin system. The solutions discussed here correspond to the half-filled case where this external field is tuned to zero.

As a consequence of the relationship (25), a soliton solution of the total particle density $\rho$ corresponds to a nonlinear excitation of the $z$-component of the spin. On the other hand, the soliton of the condensate density describes a nonlinear excitation of the inplane spin, and relates to the square of the in-plane magnetization, due to the relationship (24).

As discussed in our earlier paper [12], the existence of the two solitons in HCB has its root in the fact that both particles and holes play equal roles in deciding the dynamics of the system. It is noteworthy that the XXZ Hamiltonian (3) provides an alternative means to understand the existence of the two solitons. The spin Hamiltonian (3) contains terms with two competing effects, the easy plane anisotropy $g$ that tends to align spins in the $x y$-plane and the transverse field $h_{z}$ that favors a spin alignment along the $z$-axis. The ground state of the system consists of spins aligned on a cone that makes an angle $\theta_{0}$ with the $z$-axis where $\theta_{0}$ is determined by the background density of the atomic cloud given by $\rho_{0}=1 / 2-S_{z}^{0}=1 / 2-(1 / 2) \cos \theta_{0}$.

Interestingly, in the half-filled case $\rho^{0}=1 / 2$, the particle-hole imbalance $\delta_{0}=\left(1-2 \rho^{0}\right)$ is zero, and $h_{z}$ vanishes. In this case, the ground state consists of spins in the $x y$-plane. Therefore, the solitary wave excitation resulting in a localized change in the density makes the spins move out of the plane. In view of the fact that there is no clear preferred direction, disturbances above and below the easy plane are equally preferred. In the corresponding particle density in $\mathrm{HCB}$, the dark and antidark solitons are therefore mirror images of each other, as seen from Eq. (11). 
In contrast, as seen in Eq. (24), the condensate density of HCB is related to the square of the in plane magnetization (which maps to the GPE density profile with $\rho_{g}^{o}=1 / 4$ ) resulting in a single soliton both in the GPE and in the condensate fraction of the HCB. Such a soliton has to be a dark soliton, since the out of plane component of the spin decreases the total magnetization of the system.

In summary, soliton characteristics in the BEC of bosons in weakly and strongly interacting regimes are expected to be quite distinct. In cold atom laboratories where the interaction between atoms can be varied, one expects significant changes in the dynamics as one tunes the scattering lengths of the bosonic atoms. Therefore, our result illustrating the similarities between the solitons in these two different regimes in the special half-filled case is important. Furthermore, by exploiting the relationship between the HCB and spin systems, the connection between the GPE and HGPE solitons paves the way for relating them to magnetic solitons in a ferromagnetic spin chain with easy-plane exchange anisotropy. Thus the HGPE soliton at half-filling and the above magnetic soliton can be regarded as two other incarnations of the GPE soliton. These studies open a new way to understand and interpret many important characteristics of solitons in these systems, including the fact that it provides an intuitive understanding of why the GPE soliton is dark.

It is to be noted that it is the density in BEC that has a solitonic behavior. Thus by designing a quasi-one-dimensional optical lattice which incorporates the hard-core boson constraint of no double occupancy, and by loading them with bosonic atoms appropriately so as to simulate a half-filled lattice, it would be of interest to study soliton propagation of density in this strongly repulsive system, and investigate its relationship to the GPE dark soliton which is the hallmark of a weakly repulsive system.

\section{Note added in proof}

After the submission of this article, the collision properties of two GPE solitons and those of two HCB solitons at half-filling have been studied. These show some differences. The details will be published elsewhere.

\section{Acknowledgements}

Research of I.I.S. is supported by the grant N00014-09-1-1025A by the Office of Naval Research, and the grant 70NANB7H6138, Am 001 by the National Institute of Standards and Technology. R.B. thanks the Department of Science and Technology, India, for financial support.

\section{References}

[1] T. Dauxois, M. Peyard, Physics of Solitons, Cambridge University Press, Cambridge, 2006.

[2] M.J. Ablowitz, H. Segur, Solitons and Inverse Scattering Transform, SIAM, Philadelphia, 1981.

[3] L. Pitaevskii, S. Stringari, Bose-Einstein Condensation, Oxford University Press, Oxford, 2003.

[4] T. Tsuzuki, J. Low Temp. Phys. 4 (1971) 441.

[5] S. Burger, et al., Phys. Rev. Lett. 83 (1999) 5198; J. Denschlag, et al., Science 287 (2000) 97.

[6] E. Kolomeisky, T.J. Newman, J. Straley, X. Qi, Phys. Rev. Lett. 85 (2000) 1146.

[7] K.V. Krutitsky, J. Larson, M. Lewenstein, arXiv:0907.0625.

[8] R.V. Mishmash, I. Danshita, C.W. Clark, L.D. Carr, Phys. Rev. A 80 (2009) 053612.

[9] J.S. Langer, Phys. Rev. 167 (1968) 183.

[10] R. Balakrishnan, R. Sridhar, R. Vasudevan, Phys. Rev. B 39 (1989) 174; R. Balakrishnan, Phys. Rev. B 42 (1990) 6153.

[11] T. Matsubara, H. Matsuda, Prog. Theor. Phys. 16 (1956) 569; S. Sachdev, Quantum Phase Transitions, Cambridge University Press, Cambridge, 1999.

[12] R. Balakrishnan, I. I Satija, C.W. Clark, Phys. Rev. Lett. 103 (2009) 230403.

[13] For the definition of an antidark soliton, see Y.S. Kivshar, V.V. Afanasjev, Phys. Rev. A 44 (1991) R1446.

[14] B.B. Baizakov, et al., J. Phys. B: At. Mol. Opt. Phys. 42 (2009) 175302; G. Gligoric, et al., Phys. Rev. A 78 (2008) 063615.

[15] J.M. Radcliffe, J. Phys. A 4 (1971) 313.

[16] L.J. de Jongh, A.R. Miedema, Adv. Phys. 23 (1974) 1.

[17] A.M. Kosevich, B.A. Ivanov, A.S. Kovalev, Phys. Rep. 194 (1990) 117. 